“ (C) 2017 IEEE. Personal use of this material is permitted. Permission from IEEE must be obtained for all other uses, in any current or future media, including

reprinting/republishing this material for advertising or promotional purposes, creating new collective works, for resale or redistribution to servers or lists, or reuse of any copyrighted component of this work in other works." 


\title{
Fuzzy Integral With Particle Swarm Optimization for a Motor-Imagery-Based Brain-Computer Interface
}

\author{
Shang-Lin Wu, Member, IEEE, Tsung-Yu Hsieh, Yang-Yin Lin, Member, IEEE, Yu-Ting Liu, Chih-Yu Chen, \\ Chun-Hsiang Chuang, Member, IEEE, and Chin-Teng Lin, Fellow, IEEE
}

Abstract-A brain-computer interface (BCI) system using electroencephalography signals provides a convenient means of communication between the human brain and a computer. Motor imagery (MI), in which motor actions are mentally rehearsed without engaging in actual physical execution, has been widely used as a major BCI approach. One robust algorithm that can successfully cope with the individual differences in MI-related rhythmic patterns is to create diverse ensemble classifiers using the subband common spatial pattern (SBCSP) method. To aggregate outputs of ensemble members, this study uses fuzzy integral with particle swarm optimization (PSO), which can regulate subject-specific parameters for the assignment of optimal confidence levels for classifiers. The proposed system combining SBCSP, fuzzy integral, and PSO exhibits robust performance for offline single-trial classification of MI and real-time control of a robotic arm using MI. The main contribution of this paper is that it represents the first attempt to utilize fuzzy fusion technique to attack the individual differences problem of MI applications in real-world noisy environment. The results of this study demonstrate the practical feasibility of implementing the proposed method for real-world applications.

Index Terms-Brain-computer interface (BCI), electroencephalography (EEG), fuzzy integral, motor imagery (MI), particle swarm optimization (PSO).

B RAIN-COMPUTER interfaces (BCIs) [1] based on the user's voluntary modulations of electroencephalography (EEG) [2] signals provide an alternative method of communication between humans and machines. Despite the many pivotal

Manuscript received November 13, 2015; revised March 9, 2016; accepted May 2, 2016. Date of publication August 11, 2016; date of current version. This work was supported in part by the Aiming for the Top University Plan of National Chiao Tung University, sponsored by the Ministry of Education, Taiwan, under Grant 105W963; in part by the Cognition and Neuroergonomics Collaborative Technology Alliance Annual Program Plan, sponsored by the Army Research Laboratory under Cooperative Agreement W911NF-10-2-0022; in part by the VGHUST Joint Research Program, Tsou's Foundation, Taiwan, under Contract VGHUST105-G7-10-3; and in part by MOST104-2221-E-009-191.

S.-L. Wu and T.-Y. Hsieh are with the Institute of Electrical Control Engineering, National Chiao Tung University, Hsinchu 30010, Taiwan, R.O.C (e-mail: slwu19870511@gmail.com; aaron.eecs98@g2.nctu.edu.tw).

Y.-Y. Lin is with the Electronic Systems Research Division, National ChungShan Institute of Science and Technology, Taoyuan 32546, Taiwan, R.O.C (e-mail: oliver.yylin@gmail.com).

C.-Y. Chen is with the Brain Research Center, National Chiao Tung University, Hsinchu 30010, Taiwan, R.O.C (e-mail: abc7765kimo@ @otmail.com).

Y.-T. Liu, C.-H. Chuang, and C.-T. Lin are with the Brain Research Center, National Chiao Tung University, Hsinchu 30010, Taiwan, R.O.C, and also with the Faculty of Engineering and Information Technology, University of Technology Sydney, Sydney, NSW 2007, Australia (e-mail: tingting76319@ gmail.com; cch.chuang@gmail.com; ctlin@mail.nctu.edu.tw).

Color versions of one or more of the figures in this paper are available online at http://ieeexplore.ieee.org.

Digital Object Identifier 10.1109/TFUZZ.2016.2598362 techniques developed by the pattern recognition community that have been applied and evaluated within the context of EEGbased BCI, the overall performance of BCIs is still not robust because of inter- and intrasubject variability. This variability introduces a large number of uncertainties that severely degrade the performance of BCIs.

Among existing BCIs [3], efforts to develop EEG-based BCI systems relying on motor imagery (MI) [4] have attracted increasing attention in recent years. The brain dynamics of MI are predominantly observed in the primary sensorimotor area and resemble those observed during the actual execution of movement. A variety of feature extraction methods have been proposed to differentiate between the brain dynamics of left- and right-hand MI. In addition to event-related potentials [5], many methods [6], [7] focus on observing the difference in spectral power between the cerebral hemispheres during MI. Among the existing feature extraction methods [8]-[11], the common spatial pattern (CSP) method is one of the most effective approaches for constructing optimal spatial filters that are sensitive to differences between left and right imagery [12], [13]. However, the performance of these spatial filters depends on the operational frequency band. Searching for the optimal frequency range for each subject can be very time-consuming. To address this issue, the subband CSP (SBCSP) method [14] employs a filter bank to decompose EEG signals into different subbands as inputs to the CSP analysis. The SBCSP approach is used to extract useful features of brain activity during MI tasks; subsequently, multiple linear discriminant analysis (MLDA) [15] is applied to recognize the EEG signals in each subband spectrum. After the subband decisions are obtained from each LDA, a classifier ensemble is constructed for each subband, and a fusion algorithm is then employed to obtain a final decision. Because the decision is derived from different subband classifiers, a combination of classifiers promises to offer better uncertainty identification performance than a single classifier.

Recently, the fuzzy fusion approach [16], [17] has been shown to improve the BCI performance in terms of classification accuracy and system stationarity. One commonly used fuzzy fusion approach is fuzzy integral [18], [19], which allows the uncertain, imprecise, and incomplete information available from EEG signals to be represented and processed using the concept of fuzzy measures introduced by Sugeno [20]. This study attacks the misclassification problem that many current BCI systems experience because of variations among individuals. A judicious use of multiple sources effectively reduces individual uncertainty, and serves to enhance the reliability of the system's performance. Because the fuzzy integral [21]-[25] integrates 


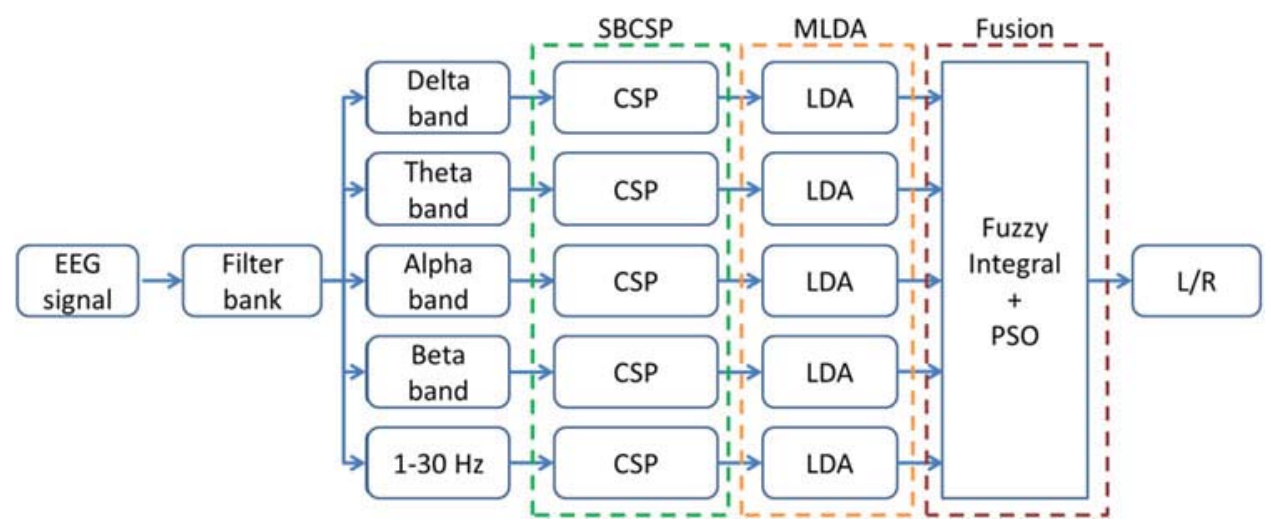

Fig. 1. System architecture of the proposed MI-based BCI fuzzy fusion.

decisions from different sources, using a combination of classifiers holds the promise of achieving better performance in uncertainty identification than the recognition technique based on the single feature. The fuzzy integral [26] is regarded as a numeric-based connective aggregation approach for obtaining collaborative decisions by integrating information from multiple classifiers.

In MI tasks, there are two main difficulties in real-world MI applications: individual difference and noisy environment. The individual differences include not only inter- but also intraindividual differences, which arise from the fact that individuals continually change over time due to factors such as fatigue, attention, and stress. Likewise, physiological signals are nonstationary and can change over time due to movement artifacts, sensor configuration, and intrinsic noise in the environment. Accordingly, features obtained from different subjects under different tempo-spatial environments might vary widely. That is, some effective features can be found in recordings from one subject but not from another. Hence, each possesses its own set of reliabilities and potential uncertainties. As a result, the performance of traditional MI systems using a single classifier to recognize all the feature usually degraded obviously under the situations of individual differences and noisy environments. To solve this problem, the proposed MI-based BCI system in this paper employs the fuzzy integral with particle swarm optimization (PSO) to classify EEG feature vectors. The fuzzy integral is a fusion technique that exploits multiple decisions from different sources to reap collaborative inferences to achieve the objectives under investigation, a result that is infeasible to achieve from each individual source separately.

In this paper, diverse LDA classifiers following the SBCSP approach are established as an ensemble of classifiers to collaboratively recognize the user's mental representation of movements from EEG patterns recorded during an MI task. Two fuzzy integral methods, i.e., the Sugeno integral [27], [28] and the Choquet integral [29], are applied to integrate the information from this ensemble of classifiers and then make a joint decision. To effectively assign confidence levels to particular classifiers, PSO [30] is employed to determine the confidence of the employed classifiers. The proposed method is demonstrated in the real-time MI control of a robotic arm.
The remainder of the paper is organized as follows. In 121 Section II, the proposed BCI for deciphering the mental re- 122 hearsal of motor actions is introduced. In Section III, an MI 123 experiment is presented. The classification results obtained us- 124 ing the proposed approach are compared with those obtained 125 using conventional ones. Finally, a brief conclusion is presented 126 and future studies are suggested in Section IV.

\section{Materials AND MethoD}

The proposed MI-based BCI system is schematically illustrated in Fig. 1. During the MI task, the EEG signals are measured by a wireless acquisition device with dry electrodes. A filter bank is then used to extract frequency components (ranging from 1 to $30 \mathrm{~Hz}$ ) from the EEG recordings. The CSP method leads to optimal variances for the discrimination of two populations of EEG related to left- and right-hand MI. Multiple LDA classifiers are established that employ CSP features to integral multiclassifiers. Finally, a fuzzy integral with PSO is then applied to fuse the decisions of classifiers and decipher the mental rehearsal of motor actions.

\section{A. EEG Acquisition Device}

The EEG acquisition device [31] was designed to measure 141 scalp EEG signals using dry electrodes [32] [see Fig. 2(a)- 142 (c)] from the sensorimotor area [see Fig. 2(d)]. The acquisition 143 device consists of a preamplifier unit, a microcontroller unit, 144 and a Bluetooth transmission unit. The wireless integrated- 145 circuit-based acquisition module has dimensions of approxi- 146 mately $55.08 \times 38.8 \times 5 \mathrm{~mm}^{3}$. The gain of the preamplifier 147 unit is set to $1361 \mathrm{~V} / \mathrm{V}$, and the cut-off frequency is regulated 148 to $0.2 \mathrm{~Hz}$ by a high-pass filter. The microcontroller unit is used 149 to regulate the signal sampling rate and for noise reduction. 150 The microcontroller unit digitizes the analog EEG signal at a 151 sampling rate of $512 \mathrm{~Hz}$. A sinc filter is used to remove frequen- 152 cies above $128 \mathrm{~Hz}$. Moreover, the ac power line noise $(60 \mathrm{~Hz}) \quad 153$ in the amplified EEG signal is reduced by the microcontroller 154 unit using a moving average. Then, the processed EEG signal is 155 transmitted to the computer using Bluetooth (v2.1+ enhanced 156 data rate). The power is supplied by a commercial $700 \mathrm{mAh} \quad 157$ $\mathrm{Li}$-ion battery, which provides over $10 \mathrm{~h}$ of operation.

\section{7} 2 3 25 27 29 130 131 132 133 134 135 136 137 138 139 0 1 3 44 45 47 49 50 1 2 54 55 56 157 


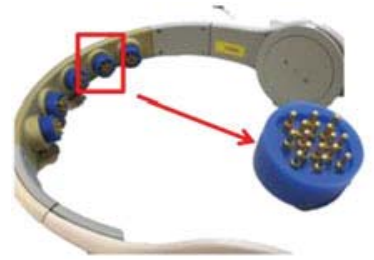

(a)

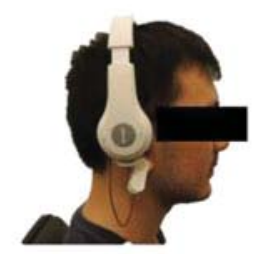

(c)

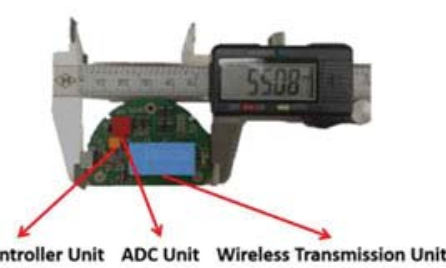

(b)

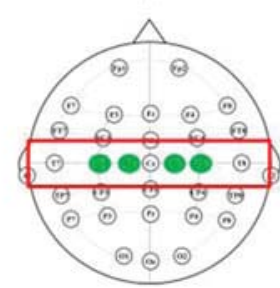

(d)
Fig. 2. Wireless and portable EEG device. (a) Dry electrodes. (b) Wireless EEG acquisition system, which consisting a preamplifier, a filter, a microcontroller, and a wireless module. Each circuit board has a width of $55.08 \mathrm{~mm}$. (c) EEG headset. (d) Placement of the four recording electrodes.

\section{B. CSP and Linear Discriminant Analysis}

Applying the proper spatial filter can improve the discrimination of data from different classes, thereby facilitating classification. The CSP approach [33] is a popular method that yields the optimal variances for the discrimination of two EEG populations related to left- and right-hand MI. In this study, the CSP method is applied to each set of filtered data $E$ to find a spatial filter matrix $W$ that maximizes the variance of the spatially filtered data of one class $\Sigma_{1}$, and simultaneously minimizes the variance of the spatially filtered data of the other class, $\Sigma_{2}$. Mathematically, the CSP criterion is written as

$$
\begin{gathered}
\text { maximize } \operatorname{tr}\left(W^{\mathrm{T}} \Sigma_{1} W\right) \\
\text { subject to } W^{\mathrm{T}}\left(\Sigma_{1}+\Sigma_{2}\right) W=I
\end{gathered}
$$

where

$$
\begin{aligned}
& \Sigma_{1}=\exp _{E_{n} \in\{\text { class } 1\}} \frac{E_{n} E_{n}^{\mathrm{T}}}{\operatorname{tr}\left(E_{n} E_{n}^{\mathrm{T}}\right)} \text { and } \\
& \Sigma_{2}=\exp _{E_{n} \in\{\text { class } 2\}} \frac{E_{n} E_{n}^{\mathrm{T}}}{\operatorname{tr}\left(E_{n} E_{n}^{\mathrm{T}}\right)} .
\end{aligned}
$$

This problem can be solved as a generalized eigenvalue problem. With the spatial filter transformation $W$ thus obtained, the spatially filtered data $Z=W^{\mathrm{T}} E$ are then used as the feature vector for LDA classifiers.

LDA [34] is a well-known binary classification method based on the estimation of the mean vectors and covariance matrices of individual classes to find the linear combination of features that maximizes the separability between distinct classes. LDA can be formulated in terms of a Bayes rule that aims to assign each sample to the class with the maximal posterior probability. In this study, multiple LDA classifiers are trained from each subband to serve as base classifiers constituting an ensemble system. The decisions derived from each LDA classifier, i.e., the posterior probabilities of left- and right-hand movements, 184 are then fused by means of a fuzzy integral.

\section{Fuzzy Integrals}

The purpose of fuzzy integral is to utilize information regarding the uncertainty or confidence of various candidate information sources during the decision-making process as represented using a fuzzy measure. For classifier fusion, an extension of the integral operator is used in the fuzzy integral to gather the objective evidence supplied by the classifiers in the form of certainty measures. Given the aforementioned benefits of this approach, the combination of classifiers based on fuzzy measures and integrals can enhance the robustness and reliability of BCI systems. In this paper, the combination of classifiers is performed by means of the Sugeno integral [27], [28] and the Choquet integral [29], which have been successfully implemented in the pattern recognition community.

The Sugeno integral is a type of integral with respect to a fuzzy measure that is defined for functions whose range is $0-1$. Given the outputs of $k$ classifiers $x_{k} \in[0,1]$, the Sugeno integral over the set $A=\left\{x_{1}, \ldots, x_{i}, \ldots, x_{k}\right\}$ of a membership function $h$ with respect to the confidence $g$ is defined as

$$
S_{g}(h)=\int_{A} h\left(x_{i}\right)^{\circ} g=\sup _{\alpha \in[0,1]}\left[\min \left(\alpha, g\left(A \cap F_{\alpha}\right)\right)\right]
$$

where $F_{\alpha}=\{x \mid h(x) \geq \alpha\}$.

The Choquet integral is another type of integral with respect to a fuzzy measure. The choice of this integral is inspired by both a theoretical property and a practical one. Specifically, it is a proper generalization of the normal integral operator. In addition, the learning task can be regarded as a convex quadratic 210 program and can therefore be solved using well-known 211 algorithms. The Choquet integral is defined as

$$
C_{g}(h)=\sum_{i=1}^{k}\left[h\left(x_{i}\right)-h\left(x_{i-1}\right)\right] g\left(A_{i}\right)
$$

where $h\left(x_{0}\right)=0$.

Note that the confidence $g$ of each classifier is heuristically 214 assigned. In this study, $g$ is proposed to be determined via PSO 215 (see Section II-D).

The joint confidence of the entire set of sources $g\left(A_{i}\right)$ can be 217 obtained as

$$
\begin{aligned}
g\left(A_{i}\right)= & g\left(\left\{h_{1}, . ., h_{i-1}\right\}\right)+g\left(\left\{h_{i}\right\}\right) \\
& +\lambda \times g\left(\left\{h_{1}, . ., h_{i-1}\right\}\right) \times g\left(\left\{h_{i}\right\}\right)
\end{aligned}
$$

where $\lambda \in(-1, \infty)$ and $\lambda$ can be obtained by solving the 219 following equation:

$$
\lambda+1=\prod_{i=1}^{k}\left(\lambda g_{i}+1\right) .
$$

Then, the final decision is determined by the class with the 221 largest fuzzy probability. 


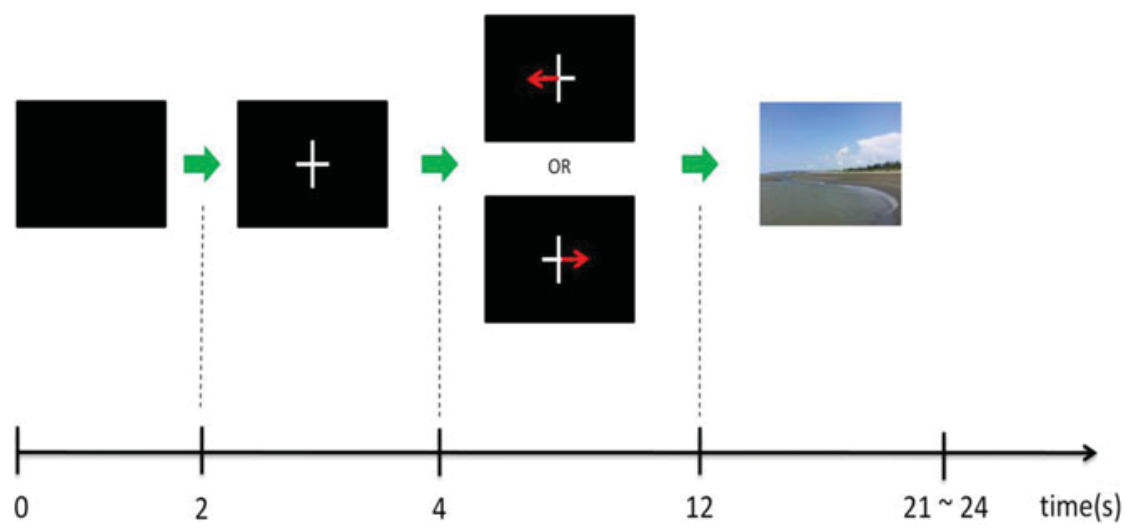

Fig. 3. Experimental paradigm.

\section{Particle Swarm Optimization}

To effectively assign confidence levels to the classifiers used in the fuzzy integral, PSO [21] is employed to update the confidence of the classifiers. The PSO algorithm is a well-known swarm intelligence technique that was developed to imitate the behavior of a flock of birds or a school of fish. The objective of PSO is to optimize a model by iteratively attempting to improve upon a candidate solution with regard to a given measure of quality. The PSO algorithm involves two critical steps, which are as follows:

1) Initialize a population of particles with a random distribution within the desired range of the search space.

2) Update the particle positions and velocities as follows:

$$
\begin{aligned}
v_{i, d} \leftarrow \omega v_{i, d} & +\phi_{p} r_{p}\left(p_{i, d}-g_{i, d}\right) \\
& +\phi_{f} r_{f}\left(f_{d}-g_{i, d}\right), \quad g_{i} \leftarrow g_{i}+v_{i}
\end{aligned}
$$

where $f$ is the best known position of the entire swarm and $p_{i, d}$ is the best known position of particle $i$. When $\omega$ is less than 1 , the particle velocities may tend toward 0 , causing the particles to fall into a local minimum and delaying convergence.

The confidential weights $g$ of the Sugeno integral and the Choquet integral are determined by PSO in this study. The initial vector that contains the fuzzy integral parameters is randomly chosen; $\omega$ is the inertial weight, $\phi_{p}$ and $\phi_{f}$ are acceleration constants, and $r_{p}$ and $r_{f}$ are random numbers drawn from the uniform distribution $\mathrm{U}(0,1)$. The confidential weights updated via PSO are calculated according to (7). When a particle finds a better position than its previous best position, the previous position is dropped and the new one is stored in the population. This value is called the personal best position of that particle, i.e., $p_{\text {best }}$. The mechanism retains a satisfactory confidential weight until the predefined number of iterations is reached. Meanwhile, the global best position, i.e., $f_{\text {best }}$, of the particle swam as a whole is updated by the particle swarm optimizer based on the particles that exist in the population. The distances between the positions of the particles and the values of $f_{\mathrm{best}}$ and $p_{\text {best }}$ decrease during optimization. This procedure allows us to search for the optimal weights for each information source to obtain an optimized output during the training phase.
III. RESULTS AND DISCUSSION

Ten male subjects, aged 22-26 years old, were recruited to 260 participate in the MI experiment. All participants were neuro- 261 logically healthy. Before the experiment, the participants were 262 required to complete an informed consent form. Each partic- 263 ipant was seated comfortably in front of a monitor, and the 264 MI task was explained via written instructions on the screen. 265 Five dry electrodes were used (four channels to record the 266 EEG signals and one for reference) to measure EEG signals 267 from the sensorimotor area. The MI experiment consisted of 268 three phases. The first phase was a baseline-constructing task 269 to establish an individual MI model of the proposed system, 270 with the aim of constructing the features for the imagery of 271 left- and right-hand movements. Twenty trials were performed 272 in this baseline-constructing phase for the imagery of both 273 left- and right-hand movements. The second phase was designed 274 to train the participants in imaging left- and right-hand move- 275 ments for EEG measurements. Each of the two directions was 276 tested 40 times. In each training trial, an arrow pointing either 277 to the left or to the right would randomly appear on the screen. 278 After each imagery trial, a picture was displayed on the screen 279 for a randomly determined period of time to help the subjects 280 relax between trials. The training phase was used to calibrate 281 the parameters of the proposed measurement system for each 282 user, with the aim of identifying each user's EEG features. The 283 last phase was the actual experiment, also with 40 MI trials per 284 direction. Upon seeing an arrow indicating a direction, the users 285 were instructed to perform imagery of the corresponding left- 286 or right-hand movement. The wireless EEG acquisition device 287 was used during the MI experiment.

\section{A. Experimental Procedure}

The experimental paradigm is illustrated in Fig. 3. A subject 290 was seated in a comfortable chair, with his hands placed on 291 a table. A blank screen was displayed for 2 s, followed by a 292 cross displayed at the center of the screen for $2 \mathrm{~s}$. Then, the 293 subject was instructed to perform left/right MI as indicated by a 294 left/right-pointing arrow, which was presented for 8 s. Finally, a 295 picture was shown on the screen for 9-12 s to allow the subject 296 to rest. 
TABLE I

ClassificAtion Results (AUC) FOR THE BASE Classifiers AND VARIOUS CONVENTIONAL AND FUZZY FUSION APPROACHES WITH FOURFOLD CROSS-VALIDATION APPLIED TEN TIMES

\begin{tabular}{lccc}
\hline \hline & \multicolumn{2}{c}{ Area Under ROC Curve } & T Test \\
\hline Single LDA & Delta LDA & $0.915 \pm 0.020$ & - \\
& Theta LDA & $0.904 \pm 0.027$ & - \\
& Alpha LDA & $0.890 \pm 0.050$ & - \\
& Beta LDA & $0.880 \pm 0.044$ & - \\
& All-band LDA & $0.900 \pm 0.040$ & - \\
\hline Conventional Methods & Voting & $0.962 \pm 0.082$ & $p<0.05$ \\
& Weighted Summation & $0.990 \pm 0.015$ & $p<0.05$ \\
& SVM & $0.993 \pm 0.022$ & $p<0.05$ \\
\hline Fuzzy Fusion & Sugeno Integral & $0.968 \pm 0.063$ & $p<0.05$ \\
& Choquet Integral & $0.992 \pm 0.014$ & $p<0.05$ \\
\hline \hline
\end{tabular}

TABLE II

CLASSIFICATION RESULTS FOR THE SUGENO INTEGRAL AND THE CHOQUET INTEGRAL AFTER PSO TRAINING WITH FOURFOLD CROSS-VALIDATION APPLIED TEN TIMES

\begin{tabular}{lccc}
\hline \hline & Fuzzy Fusion & w/o PSO & w/ PSO \\
\hline Fuzzy Fusion & Sugeno & $0.968 \pm 0.063$ & $\mathbf{0 . 9 9 8} \pm \mathbf{0 . 0 4 0}$ \\
& Choquet & $0.992 \pm 0.014$ & $\mathbf{0 . 9 9 8} \pm \mathbf{0 . 0 0 3}$ \\
\hline
\end{tabular}

\section{B. Fuzzy Fusion Performance}

In MLDA, classifiers are constructed using a combination of features from multiple frequency bands, including four separate frequency bands (i.e., the delta, theta, alpha, and beta bands) and the full-band signal ranging from 1 to $30 \mathrm{~Hz}$. In each frequency band, an LDA classifier is constructed using features extracted via CSP projection. Consequently, the MLDA is established using the spatial pattern features from these five frequency bands. The separate frequency bands provide the features of each band in greater detail and allow more features to be obtained. Accordingly, the Sugeno integral or the Choquet integral is used for fuzzy fusion to integrate the MLDA decisions constructed using the five base classifiers, namely, the delta, theta, alpha, beta, and all-band LDA classifiers, in the proposed system. After the aggregation of the results from different bands, the fuzzy fusion mechanism is applied to make the final decision. Initially, the weights of each classifier in the Sugeno integral and the Choquet integral are all set to 0.2. The PSO algorithm is later applied to update these weights.

The performances of the two fuzzy integrals and of several conventional fusion methods were evaluated in terms of the area under the ROC curve (AUC). As shown in Table I, each fusion technique outperformed each single classifier, with the proposed fusion architecture yielding not only higher AUC values but also smaller standard deviations. In comparison with existing fusion techniques, the weighted summation approach, the support vector machine (SVM) approach [35], and the Choquet integral outperformed the voting approach [36] and the Sugeno integral. As shown in Table II, after the application of PSO to update the weights of the classifiers, the results of both the Sugeno and Choquet integrals exhibited improvements, from $0.968 \pm 0.063$ to $0.998 \pm 0.040$ and from $0.992 \pm 0.014$ to $0.998 \pm 0.003, \quad 329$ respectively. The AUC was improved and the standard devia- 330 tion was reduced, indicating that the system achieved higher 331 accuracy and better stability.

\section{Proposed Online BCI System and Its Application}

The flow chart for a subsequent online experiment is shown 334 in Fig. 4. The offline experiment reported above was initially 335 required for advance model generation. The models thus gen- 336 erated could subsequently be applied in an online experiment 337 using the proposed BCI system. When performing the online 338 experiment, each subject wore an EEG acquisition system on 339 the top of his head along the central sulcus, and the reference 340 was recorded at the earlobes on both sides. Each subject was 341 required to perform a full experiment consisting of four sessions 342 (160 trials), and the model previously derived for that subject 343 was applied in the online system.

In each trial, the user interface of the online system presented a randomly generated cue, namely, an arrow pointing to the left or to the right at the center of the screen. Each classification result was recorded as a score of +1 or -1 ; the total accumulated score was calculated after every trial. If the final score was above +25 or below -25 , the system made a final decision of either a left command or a right command, respectively. Because the computing speed of the online system was $25 \mathrm{~Hz}$, if the subject wished to issue a left or right command, he was required to continuously think about the same direction for $1 \mathrm{~s}$. After each trial, the classification result accumulated over $1 \mathrm{~s}$ was plotted as a bar. The accuracy rate was recorded at the top of the window. The processing time (from the input of the raw data to the output of the result) was $40.1715 \mathrm{~ms}$, as shown in Fig. 5. In other words, this system is capable of computing at a rate of approximately $25 \mathrm{~Hz}$ when performing online computations. This computation rate was the basis for the selection of a value of 25 points as the threshold for the online interface. The accuracy rate achieved in the online test was approximately $86 \%$. Depending on the classification result, a robotic arm would immediately grasp a glass to either the left or the right. The robotic arm used in this experiment is commercially available on the rehabilitation market (Kinova, Canada). It consists of a six-axis robotic manipulator arm with a three-fingered hand. This robotic arm can perform a wide variety of functions with graceful movements.

\section{Reliability Test}

A further test was performed to confirm the model reliability. 371 In this test, the performance of the algorithm was evaluated us- 372 ing data acquired from the same subject but on a different day. 373 The training set included data recorded continuously from four 374 experimental sessions (160 trials) in a single day for one sub- 375 ject. The test set included data from two experimental sessions 376 (80 trials) recorded on a different day for the same subject. Af- 377 ter a model was generated from the training set, that model was 378 applied to the test data to evaluate its performance. The accu- 379 racy rate of prediction was found to be $91.25 \%$, indicating good 380 model stability. (t)

\section{4} 345 346 347 348 349 350 351 352 353 354 355 356 357 358 359 360 361 362 363 364 365 366 367 368 369 381

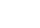

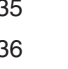
(n) . to , . .

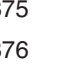

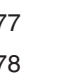
(t) 


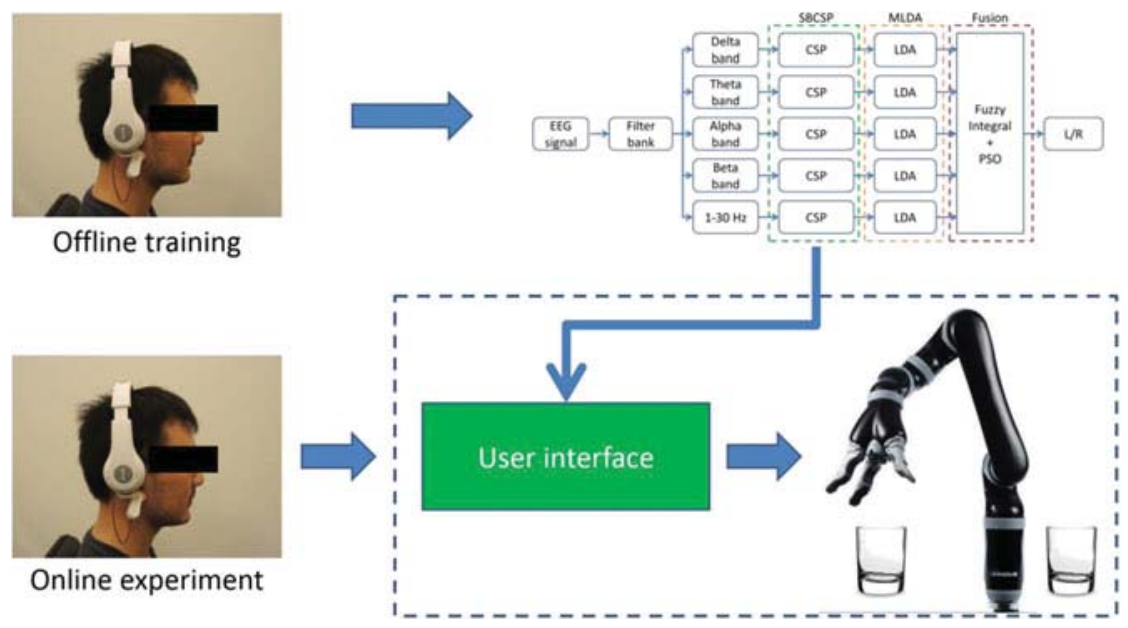

Fig. 4. Flow chart of the proposed MI-based BCI system application.

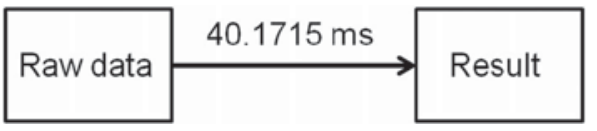

Fig. 5. Signal processing time within the proposed online system.

\section{CONCLUSION}

In this study, we propose an innovative ensemble method with swarm-optimized fuzzy integral for an MI recognition task. The fuzzy integral provides an effective mechanism for representing and processing the uncertainty of the outputs of individual ensemble members using the concept of fuzzy measures. Furthermore, PSO is used to update the confidence of the employed classifiers. The experimental results derived from a typical MI task show that the best classification accuracy is achieved when applying the Choquet integral with PSO training in the fusion phase. Additionally, the results demonstrate the feasibility of implementing the proposed system in real-time robotic arm control. In the future, developing a more advanced BCI system with fuzzy theory will be necessary to enable the execution of multidirectional movements.

\section{ACKNOWLEDGMENT}

The authors would like to thank Prof. J.-Y. Chang and all the members of the Brain Research Center, National Chiao Tung University, Taiwan.

\section{REFERENCES}

[1] G. E. Fabiani, D. J. McFarland, J. R. Wolpaw, and G. Pfurtscheller, "Conversion of EEG activity into cursor movement by a brain-computer interface (BCI)," IEEE Trans. Neural. Syst. Rehabil. Eng., vol. 12, no. 3, pp. 331-338, Sep. 2004.

[2] S. Ferdowsi, S. Sanei, V. Abolghasemi, J. Nottage, and O. O'Daly, "Removing ballistocardiogram artifact from EEG using short- and long-term linear predictor," IEEE Trans. Biomed. Eng., vol. 60, no. 7, pp. 1900-1911, Jul. 2013.

[3] M. Bamdad, H. Zarshenas, and M. A. Auais, "Application of BCI systems in neurorehabilitation: A scoping review," Disability Rehabil. Assistive Technol., vol. 10, pp. 355-364, 2015.
[4] T. Mulder, "Motor imagery and action observation: Cognitive tools 413 for rehabilitation," J. Neural Transmiss., vol. 114, pp. 1265-1278, 414 2007.

[5] S. S. Chung, L. K. McEvoy, M. E. Smith, A. Gevins, K. Meador, and 416 K. D. Laxer, "Task-related EEG and ERP changes without performance 417 impairment following a single dose of phenytoin," Clin. Neurophysiol., 418 vol. 113, pp. 806-814, 2002.

[6] J. M. Cano-Izquierdo, J. Ibarrola, and M. Almonacid "Improving mo- 420 tor imagery classification with a new BCI design using neuro-fuzzy S- 421 dFasArt," IEEE Trans. Neural. Syst. Rehabil. Eng., vol. 20, no. 1, pp. 2-7, 422 Jan. 2012.

[7] Y.H. Liu, C. T. Wu, Y. H. Kao, and Y. T. Chen, "Single-trial EEG-based emotion recognition using kernel Eigen-emotion pattern and adaptive sup- 425 port vector machine," in Proc. 35th Annu. Int. Conf. IEEE Eng. Med. Biol. 426 Soc., Osaka, Japan, 2013, pp. 4306-4309.

[8] V. Bostanov, "BCI competition 2003-Data sets Ib and IIb: Feature ex- 428 traction from event-related brain potentials with the continuous wavelet 429 transform and the t-value scalogram," IEEE Trans. Biomed. Eng., vol. 51, 430 no. 6, pp. 1057-1061, Jun. 2004. weighted wavelet transform features," J. Neurosci. Methods, vol. 176, 433 no. 2, pp. 310-318, 2009.

[10] B. Obermaier, C. Neuper, C. Guger, and G. Pfurtscheller, "Information 4 transfer rate in a five-classes brain-computer interface," IEEE Trans. Neu- 436 ral Syst. Rehabil. Eng., vol. 9, no. 3, pp. 283-288, Sep. 2001.

[11] D. P. Burke, S. P. Kelly, P. de Chazal, R. B. Reilly, and C. Finucane, 438 "A parametric feature extraction and classification strategy for brain- 439 computer interfacing," IEEE Trans. Neural. Syst. Rehabil. Eng., vol. 13, 440 no. 1, pp. 12-17, Mar. 2005.

[12] R. Zhang et al. "Control of a wheelchair in an indoor environment based 442 on a brain-computer interface and automated navigation," IEEE Trans. 443 Neural Syst. Rehabil. Eng., vol. 24, no. 1, pp. 128-139, Jan. 2016.

[13] P. Wei, W. He, Y. Zhou, and L. Wang, "Performance of motor imagery 445 brain-computer interface based on anodal transcranial direct current stim- 446 ulation modulation," IEEE Trans. Neural Syst. Rehabil. Eng., vol. 21, 447 no. 3, pp. 404-415, May 2013.

[14] Q. Novi, G. Cuntai, T. H. Dat, and X. Ping, "Sub-band common spatial pat- 449 tern (SBCSP) for brain-computer interface," in Proc. 3rd Int. IEEE/EMBS 450 Conf. Neural Eng., 2007, pp. 204-207.

[15] R. Zhang, P. Xu, L. Guo, Y. Zhang, P. Li, and D. Yao, "Z-score linear 452 discriminant analysis for EEG based brain-computer interfaces," PLoS 453 One, vol. 8, pp. 1-7, 2013.

[16] G. Salimi-Khorshidi, A. M. Nasrabadi, and M. H. Golpayegani, "Fusion 455 of classic P300 detection methods' inferences in a framework of fuzzy 456 labels," Artif Intell. Med., vol. 44, pp. 247-259, 2008. X. Luqiang and X. Guangcan, "Study on power spectrum signal 458 fuzzy fusion for motor imagery," Comput. Eng., vol. 41, pp. 306-309, 459 2015

[18] B. S. Yoo and J. H. Kim, "Fuzzy integral-based gaze control of a robotic 461 head for human robot interaction," IEEE Trans. Cybern., vol. 45, no. 9, 462 pp. 1769-1783, Sep. 2015.

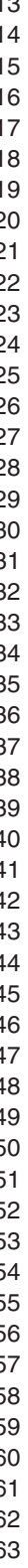

5


[19] J. K. Yoo and J. H. Kim, "Fuzzy integral-based gaze control architecture incorporated with modified-univector field-based navigation for humanoid robots," IEEE Trans. Syst. Man, Cybern. B, Cybern., vol. 42, no. 1, pp. 125-139, Feb. 2012.

[20] T. Murofushi and M. Sugeno, "A theory of fuzzy measures: Representations, the Choquet integral, and null sets," J . Math. Anal . Appl., vol. 159, pp. 532-549, 1991.

[21] F. Cavrini, L. Bianchi, L. R. Quitadamo, and G. Saggio, "A fuzzy integral ensemble method in visual P300 brain-computer interface," J. Comput. Intell. Neurosci., vol. 2016, 2016, Art. no. 49.

22] Z. Shoaie Shirehjini, S. Bagheri Shouraki, and M. Esmailee, Variant Combination of Multiple Classifiers Methods for Classifying the EEG Signals in Brain-Computer Interface . Berlin, Germany: Springer, 2009.

[23] M. Grabisch, H. T. Nguyen, and E. A. Walker, Fundamentals of Un certainty Calculi with Applications to Fuzzy Inference, vol. 30. Berlin, Germany: Springer, 1995.

[24] M. K. Wali, M. Murugappan, and B. Ahmad, "Subtractive fuzzy classifier based driver distraction levels classification using EEG," J. Phys. Therapy Sci., vol. 25, pp. 1055-1058, Sep. 2013.

[25] J. W. Bang, J. S. Choi, H. Heo, and K. R. Park, "A fuzzy-based fusion method of multimodal sensor-based measurements for the quantitative evaluation of eye fatigue on 3D displays," Sensors (Basel), vol. 15, pp. 10825-10851, 2015.

26] Q. Gao, L. Liu, G. Feng, and Y. Wang, "Universal fuzzy integral slidingmode controllers for stochastic nonlinear systems," IEEE Trans. Cybern., vol. 44, no. 12, pp. 2658-2669, Dec. 2014

[27] M. F. Anderson, D. T. Anderson, and D. J. Wescott, "Estimation of adult skeletal age-at-death using the Sugeno fuzzy integral," Amer. J. Phys. Anthropol., vol. 142, pp. 30-41, 2010.

[28] H. Agahi, R. Mesiar, and Y. Ouyang, "On some advanced type inequalities for Sugeno integral and T-(S-)evaluators," Info. Sci., vol. 190, pp. 64-75, 2012.

29] M. Singh, V. K. Madasu, S. Srivastava, and M. Hanmandlu, "Choquet fuzzy integral based verification of handwritten signatures," J. Intell. Fuzzy Syst., vol. 24, pp. 145-161, 2013.

[30] C. Liu, W.-B. Du, and W.-X. Wang, "Particle swarm optimization with scale-free interactions," PLOS ONE, vol. 9, pp. 1-8, 2014.

31] L. D. Liao et al., "A novel 16-channel wireless system for electroencephalography measurements with dry spring-loaded sensors," IEEE Trans. Instrum. Meas., vol. 63, no. 6, pp. 1545-1555, Jun. 2014.

[32] C. T. Lin, L. D. Liao, Y. H. Liu, I. J. Wang, B. S. Lin, and J. Y. Chang, "Novel dry polymer foam electrodes for long-term EEG measurement," IEEE Trans. Biomed. Eng., vol. 58, no. 5, pp. 1200-1207, May 2011.

[33] K. Yu, K. Shen, S. Shao, W. C. Ng, and X. Li, "Bilinear common spatial pattern for single-trial ERP-based rapid serial visual presentation triage," J. Neural Eng., vol. 9, pp. 1-8, 2012.

[34] H. P. Hsu and J. S. Shih, "Multi-channel surface acoustic wave sensors based on principal component analysis (PCA) and linear discriminate analysis (LDA) for organic vapors," J. Chin. Chem. Soc., vol. 53, pp. 815-824, 2006.

[35] P. J. Facchini and B. St-Pierre, "Synthesis and trafficking of alkaloid biosynthetic enzymes," Curr. Opin. Plant Biol., vol. 8, pp. 657-666, Dec. 2005.

[36] M. Almonacid, J. Ibarrola, and J. M. Cano-Izquierdo, "Voting strategy to enhance multimodel EEG-based classifier systems for motor imagery BCI,’ IEEE Syst. J., vol. 10, pp. 1082-1088, 2016.

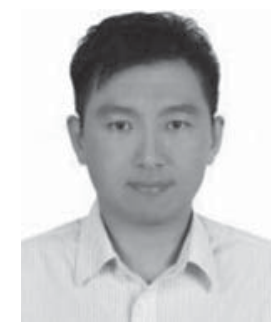

Yu-Ting Liu received the B.S. degree from the De- 532 partment of Electrical and Control Engineering, Na- 533 tional Chiao-Tung University, Hsinchu, Taiwan, in 534 2010. He is currently working toward the Ph.D. 535 degree in the Institute of Electrical and Control 536 Engineering, National Chiao Tung University. 537

His current research interests include fuzzy logic 538 theory, machine learning, computer vision, and EEG 539 analysis and application.

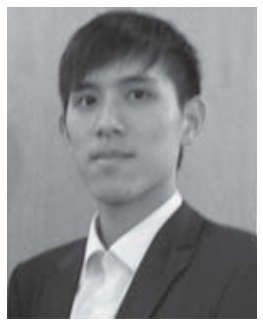

Tsung-Yu Hsieh received the B.S. degree in elec- 542 trical engineering and computer science, in 2013, 543 and the M.S. degree from the Department of Elec- 544 trical and Control Engineering, National Chiao-Tung 545 University, Hsinchu, Taiwan, in 2015. He is currently 546 working toward the Ph.D. degree at the Department 547 of Computer Science and Engineering, Pennsylvania 548 State University.

His current research interests include fuzzy logic 550 theory, machine learning, computer vision, and EEG 551 analysis and application.

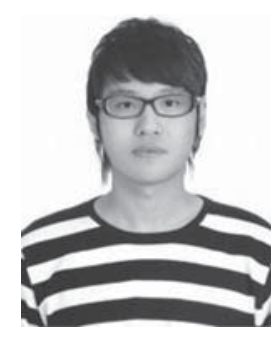

Yang-Yin Lin (M'13) received the M.S. degree 554 from the Institute of Electrical Engineering, National 555 Chung Hsing University, Taichung, Taiwan, in 2008, 556 and the Ph.D. degree from the Department of Elec- 557 trical Engineering, National Chiao Tung University, 558 Hsinchu, Taiwan, in 2013.

$\mathrm{He}$ is currently working as a Researcher Engi- 560 neer at the Electronic Systems Research Division, 561 National Chung-Shan Institute of Science and Tech- 562 nology, Taoyuan, Taiwan. His current research inter- 563 ests include evolutionary computation, type-2 neural 564 fuzzy systems, deep learning, transfer fuzzy learning, and field-programmable 565 gate array chip design based on neural network architecture.

Shang-Lin Wu (M'15) was born in Kaohsiung, Taiwan, in 1987. He received the B.S. and M.S. degrees from the Department of Computer Science and Information Engineering, National Chiayi University, Chiayi, Taiwan, in 2009 and 2011, respectively. He is currently working toward the Ph.D. degree in the Institute of Electrical Control Engineering, National Chiao Tung University, Hsinchu, Taiwan.

His research interests include fuzzy theory, transfer learning, biomedical signal processing, biofeedback control, and embedded system.

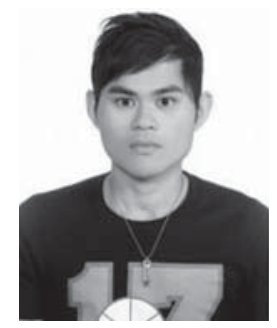

Chih-Yu Chen was born in Kaohsiung, Taiwan, 568 R.O.C., on March 20, 1988. He received the M.S. 569 degree from the Insitute of Biomedical Engineering, 570 National Chiao Tung University, Hsinchu, Taiwan, 571 in 2013 .

$\mathrm{He}$ is currently with the Quanta Computer 573 Incorporation, Taoyuan, Taiwan. 574 


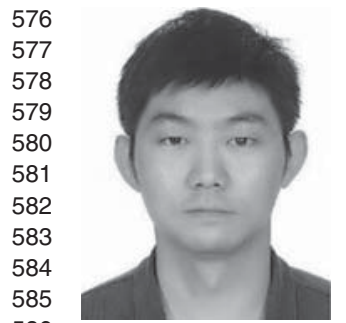

Chun-Hsiang Chuang (M'16) received the B.S. degree in mathematics education from Taipei Municipal Teachers College, Taipei, Taiwan, in 2004, the M.S. degree in educational measurement and statistics from National Taichung University, Taichung, Taiwan, in 2009, and the Ph.D. degree in electrical engineering from National Chiao Tung University (NCTU), Hsinchu, Taiwan, in 2014.

He was a Visiting Scholar at Swartz Center for Computational Neuroscience, University of California, from 2012 to 2013 . He is currently a Postdoctoral Researcher of Brain Research Center at NCTU. His research interests include machine learning, computational neuroscience, biomedical signal processing, and brain-computer interface.

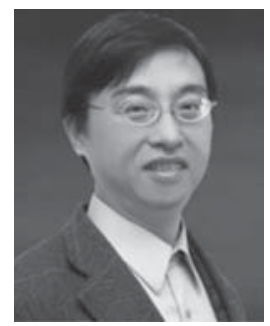

Chin-Teng Lin (F'05) received the B.S. degree from 591 National Chiao-Tung University (NCTU), Hsinchu, 592 Taiwan, in 1986, and the Master's and Ph.D. de- 593 grees in electrical engineering from Purdue Univer- 594 sity, West Lafayette, IN, USA in 1989 and 1992, 595 respectively.

He is currently the Chair Professor of Faculty of 597 Engineering and Information Technology, University 598 of Technology Sydney, Sydney, NSW, Australia, the 599 Chair Professor of Electrical and Computer Engineer- 600 ing, NCTU, International Faculty of the University of 601 California at San-Diego, and Honorary Professorship of the University of Not- 602 tingham, Nottingham, U.K. He has published more than 200 journal papers 603 (Total Citation: 20 155, H-index: 53, i10-index: 373) in the areas of neural 604 networks, fuzzy systems, multimedia hardware/software, and cognitive neuro- 605 engineering, including approximately 101 IEEE journal papers. 606

Dr. Lin was promoted as an International Fuzzy Systems Association Fellow 607 in 2012. Since 2011, he has been elected as the Editor-in-Chief of the IEEE 608 TRANSACTIONS ON FUZZY SYSTEMS. He also served on the Board of Gover- 609 nors at the IEEE Circuits and Systems (CAS) Society in 2005-2008, the IEEE 610 Systems, Man, Cybernetics Society in 2003-2005, the IEEE Computational In- 611 telligence Society (CIS) in 2008-2010, and the Chair of the IEEE Taipei Section 612 in 2009-2010. He is the Distinguished Lecturer of the IEEE CAS Society from 613 2003 to 2005, and the CIS Society from 2015-2017. He served as the Deputy 614 Editor-in-Chief of the IEEE TRANSACTIONS ON CIRCUITS AND SYSTEMS-II in 615 2006-2008. He was the Program Chair of the IEEE International Conference 616 on Systems, Man, and Cybernetics in 2005 and the General Chair of the 2011617 IEEE International Conference on Fuzzy Systems. He is the co-author of Neural 618 Fuzzy Systems (Prentice-Hall), and the author of Neural Fuzzy Control Systems 619 with Structure and Parameter Learning (World Scientific). 